\title{
Performance of Cardiovascular Risk Equations versus Vascular Ultrasound in Systemic Lupus Erythematosus in a Black African Population
}

\author{
Sow Maïmouna1 ${ }^{*}$, Kane Baïdy Sy${ }^{1}$, Diouf Marguerite Téning2, Aw Fatou'2, Ndao Awa Cheikh¹, \\ Samba Abdourahmane ${ }^{3}$, Ndiaye Mouhamadou Bamba ${ }^{2}$, Ndongo Souhaibou ${ }^{4}$, Diao Maboury², \\ Pouye Abdoulaye ${ }^{1}$
}

\author{
${ }^{1}$ Internal Medecine Department of Aristide Le Dantec Teaching Hospital of Dakar, Senegal \\ ${ }^{2}$ Cardiology Department of Aristide Le Dantec Teaching Hospital of Dakar, Senegal \\ ${ }^{3}$ Biochemial Department of Aristide Le Dantec Teaching Hospital of Dakar, Senegal \\ ${ }^{4}$ Internal Medecine Department of Dalal Jamm Hospital of Dakar, Senegal \\ Email: *mouna89sow@hotmail.fr
}

How to cite this paper: Maïmouna, S., Sy, K.B., Téning, D.M., Fatou, A., Cheikh, N.A., Abdourahmane, S., Bamba, N.M., Souhaibou, N., Maboury, D. and Abdoulaye, P. (2021) Performance of Cardiovascular Risk Equations versus Vascular Ultrasound in Systemic Lupus Erythematosus in a Black African Population. World Journal of Cardiovascular Diseases, 11, 523-532. https://doi.org/10.4236/wjcd.2021.1111049

Received: October 20, 2021

Accepted: November 27, 2021

Published: November 30, 2021

Copyright () 2021 by author(s) and Scientific Research Publishing Inc. This work is licensed under the Creative Commons Attribution International License (CC BY 4.0).

http://creativecommons.org/licenses/by/4.0/ (c) (i) Open Access

\begin{abstract}
Introduction: Cardiovascular risk is increased in systemic lupus erythematosus. Cardiovascular events are the first cause of death in lupus after five years duration. Prevention of cardiovascular events needs a good evaluation of the risk. In this work, we tried to evaluate the performance of conventional and adjusted forms of cardiovascular risk equations to predict high risk in lupus patients, in comparison with carotid ultrasound. Method: We realized a cross-sectional study during the period from 24 August 2017 to 22 November 2018. Consenting patient meeting the 1997 American college of Rheumatology criteria of systemic lupus erythematosus were recruited. The clinical characteristics and the treatment data were informed. Traditional cardiovascular risk factors were also investigated, and the assessment of cardiovascular risk was performed by Framingham and SCORE equations and their modified forms (multiplication by a factor of 1.5). Carotid ultrasound was used to detect atherosclerosis by measuring intima media thickness and searching for carotid plaques. In last, we compared cardiovascular risk levels by equations to results of carotid ultrasound. Statistical analysis and data collection were performed using SPSS 23.0 software. Results: Forty-nine patients with a sex ratio of 0.13 and a mean age of $33.5( \pm 11.3)$ years were enrolled. More than half of patients had dyslipidemia. More than $80 \%$ of the population were at low cardiovascular risk according to the equations. However, $16 \%$ of cases had carotid atherosclerosis. Between $50 \%$ and $100 \%$ of patients having atherosclerosis were considered at low or moderate risk by equations. The best
\end{abstract}


sensitivity to predict cardiovascular risk was given by modified Framingham (50\%). Conclusion: In our study, conventional and modified risk equations had a bad performance to predict cardiovascular risk in systemic lupus erythematosus.

\section{Keywords}

Cardiovascular Risk Equations, Carotid Ultrasound, Systemic Lupus Erythematosus

\section{Introduction}

Systemic lupus erythematosus (SLE) is the prototype of systemic autoimmune diseases. Its course is characterized by two mortality peaks, one early related to disease activity and infectious complications and the other late (beyond 5 years) secondary to cardiovascular events. Cardiovascular mortality is estimated to account for $10 \%-20 \%$ of overall mortality in SLE [1]. The risk of cardiovascular events is increased (50-fold) during SLE. Almost one-third of patients have carotid plaques [2]. It is therefore important to be able to assess the risk accurately to better prevent cardiovascular events [3]. In general population, it is possible to estimate the absolute coronary and cerebrovascular risk in each patient, by using equations established. The most used are Framingham and Systematic Coronary Risk Evaluation (SCORE). All the same, these equations have limits. Indeed, their predictive value is imprecise in young subjects, they do not take into account certain risk factors and their geographic validity is not clear [4] [5]. In SLE, inflammation which is the promoting factor for atherosclerosis is not taken into account by the risk equations. There is a very strong correlation between coronary atherosclerosis and ultrasound measurement of carotid intima-media thickness. In the general population, intima-media thickness and carotid plaque are independent prognostic markers of the risk of cardiovascular events [6]. Noninvasive ultrasound methods can detect atherosclerosis in $28 \%-40 \%$ of lupus patients and therefore constitute in this sense an objective and efficient tool for prejudging the vascular risk of patients [7].

A study in American population has shown that cardiovascular diseases related to atherosclerosis in SLE are more frequent in black subjects [8]. However, the cardiovascular risk of SLE remains poorly studied in sub-Saharan Africa.

Therefore, we conducted this study with the objective of evaluating the performance of cardiovascular risk equations in predicting this risk in SLE compared to vascular ultrasound in defining high cardiovascular risk.

\section{Method}

Our study took place at the Aristide Le Dantec teaching hospital in Dakar in the internal medicine, cardiology and biochemistry departments. It was a descriptive and analytical cross-sectional study done during the period from 24 August 2017 
to 22 November 2018. All consenting patients meeting the 1997 American college of Rheumatology (ACR) criteria of SLE were included consecutively [9]. Patients with chronic kidney disease, HIV infection or history of cardiovascular events were not included.

The clinical characteristics of SLE, as time to diagnosis, all clinical abnormalities related to the disease, and the disease activity assessed by the clinical Systemic Lupus Erythematosus Disease Activity Index (cSLEDAI) were recorded from their medical file [10]. Treatment data (duration, dosage and cumulative dose of corticosteroids, background therapy and other treatments) were specified. The classical cardiovascular risk factors as age, gender, diabetes, hypertension, smoking, physical inactivity, overall and abdominal obesity and dyslipidemia were investigated. The assessment of cardiovascular risk was performed by the Framingham and SCORE equations and their modified forms (SCORE/m and Framingham/m) adjusted (multiplication by a factor 1.5).

The definition of different levels of risk is presented in Table 1.

Vascular ultrasound was performed by a cardiologist using a device with a linear probe with a frequency of 3 to $13 \mathrm{Mhz}$. Measurement of carotid intima-media thickness (cIMT) was performed on the common carotid artery at $1.5 \mathrm{~cm}$ from the bulb using an automated method. Vascular ultrasound also detected the existence of carotid plaques. Carotid atherosclerosis was defined by a cIMT $\geq 0.9$ $\mathrm{mm}$ and/or the presence of carotid plaques [11]. In last, we determined the proportion in which patients with carotid atherosclerosis were classified as high or very high risk. In the same way, we searched the proportion of patients having carotid atherosclerosis but classified at low or moderate risk.

Statistical analysis and data collection were performed using SPSS 23.0 software. Quantitative variables were expressed as mean plus or minus standard deviation and qualitative variables as number and percentage. The results were presented in tables.

\section{Results}

\section{Characteristics of our study population}

We recruited 49 patients with a sex ratio of 0.13 and a mean age of 33.5 $( \pm 11.3)$ years. Cutaneous and hematological manifestations were predominant, followed by rheumatological and renal involvement. Serous and neurological manifestations were rarer. The disease was active. Most of patients were on

Table 1. Interpretation of the values of Framingham, SCORE and their modified forms.

\begin{tabular}{ccc}
\hline Cardiovascular level risk & Framingham/Framingham $/ \mathrm{m}$ & SCORE/SCORE $/ \mathrm{m}$ \\
\hline Very high risk & $>20 \%$ & $>15 \%$ \\
High risk & $15 \%-20 \%$ & $5 \%-15 \%$ \\
Moderate risk & $10 \%-15 \%$ & $1 \%-5 \%$ \\
Low risk & $5 \%-10 \%$ & $<1 \%$
\end{tabular}


Very low risk $\leq 5 \%$

corticosteroids and hydroxychloroquine. Cyclophosphamide was the most immunosuppressive used followed by azathioprine. In Table 2, socio-demographic and clinical data of the patients are summarized.

\section{Traditional cardiovascular risk factors}

Diabetes, hypertension, smoking, and sedentary lifestyle were noted in 2, 6 and 3 patients respectively. Obesity was present in 4 cases and 3 patients met the definition of metabolic syndrome. Dyslipidemia was found in more than half of the cases $(63.26 \%)$. More than a quarter (26.53\%) had low-HDL cholesterolemia. High triglyceridemia was found in $34.6 \%$ of cases. Hyperuricemia was noted in $48 \%$ of patients. Renal insufficiency was noted in 4 patients.

\section{Cardiovascular risk equations}

Most of our study population (more than $80 \%$ ) was classified at low cardiovascular risk according to the risk equations.

The results of the cardiovascular risk assessment according to the Framingham and SCORE equations and their modified version are shown in Table 3.

Carotid atherosclerosis

The mean cIMT was $0.587( \pm 0.15) \mathrm{mm}$ and carotid atherosclerosis with carotid

Table 2. characteristics of our study population.

\begin{tabular}{lc}
\hline Socio-demographic and clinical data & Number \\
\hline Sex ratio M/F & $6 / 43(0.13)$ \\
Age mean (years) & $33.5( \pm 11.3)$ \\
Disease duration (month) & $60( \pm 59.3)$ \\
Diagnostic delay (month) & $30.16( \pm 39.2)$ \\
Clinical manifestations & \\
Cutaneous & $32(65 \%)$ \\
Rheumatology & $21(42 \%)$ \\
Hematology & $31(63 \%)$ \\
Renal & $20(40 \%)$ \\
Neurology & $5(10 \%)$ \\
Serous & $4(8 \%)$ \\
Disease activity & $6.59( \pm 5.6)$ \\
Treatment data & \\
Corticosteroids & $46(93 \%)$ \\
Duration (month) & $36.46( \pm 53.4)$ \\
Daily dose (mg/day) & $22.61( \pm 18.9)$ \\
Cumulative dose (mg) & $894.94( \pm 2618.4)$ \\
Hydroxychloroquine & $48(97 \%)$ \\
& \\
&
\end{tabular}


Table 3. Cardiovascular risk level according to the Framingham and SCORE equations and their modified versions.

\begin{tabular}{ccccc}
\hline $\begin{array}{c}\text { Cardiovascular } \\
\text { risk level }\end{array}$ & $\begin{array}{c}\text { Framingham } \\
\text { n (\%) }\end{array}$ & $\begin{array}{c}\text { Adjusted } \\
\text { Framingham n (\%) }\end{array}$ & $\begin{array}{c}\text { SCORE } \\
\text { n (\%) }\end{array}$ & $\begin{array}{c}\text { Adjusted } \\
\text { sCORE n (\%) }\end{array}$ \\
\hline Very low risk & $40(82 \%)$ & $37(75 \%)$ & $\mathrm{NA}^{*}$ & $\mathrm{NA}^{\star}$ \\
Low risk & $6(12 \%)$ & $4(8 \%)$ & $46(94 \%)$ & $44(90 \%)$ \\
Moderate risk & $2(4 \%)$ & $5(10 \%)$ & $3(6 \%)$ & $4(8 \%)$ \\
High risk & $1(2 \%)$ & $3(6 \%)$ & 0 & $1(2 \%)$ \\
Very high risk & 0 & 0 & 0 & 0 \\
\hline
\end{tabular}

$\mathrm{NA}=$ no applicable.

Table 4. Carotid ultrasound findings in lupus patients.

\begin{tabular}{cc}
\hline Carotid Atherosclerosis & Systemic lupus erythematosus \\
\hline IMT mean $(\mathrm{mm})$ & 0.587 \\
IMT mean right $(\mathrm{mm})$ & 0.587 \\
IMT mean left $(\mathrm{mm})$ & 0.592 \\
IMT $\geq 0.9 \mathrm{~mm} \mathrm{n}(\%)$ & $2(4 \%)$ \\
Plaques $\mathrm{n}(\%)$ & $8(16 \%)$ \\
\hline
\end{tabular}

$\mathrm{IMcT}=$ carotid intima média thickness.

plaques was present in 8 patients (16\%).

Table 4 summarizes the vascular ultrasound data of our study population.

Performance of risk equations in predicting high cardiovascular risk in lupus

Classical equations underestimated the cardiovascular risk. In fact, between $75 \%$ and $100 \%$ of patients having carotid atherosclerosis were classified in moderate or low cardiovascular risk by this equation. Only between 0 to $25 \%$ of patients with carotid atherosclerosis were considered at very high or high cardiovascular risk. In the same way, the adjusted equation underestimated also the risk. From $50 \%$ to $87.5 \%$ patient classified at moderate or low cardiovascular risk had carotid atherosclerosis. The modified SCORE had a bad sensibility $(12.5 \%)$ and the modified Framingham offered the best sensibility (50\%) in atherosclerosis prediction. In contrast, their specificity was very good because from $90 \%$ to $100 \%$ of patients having carotid atherosclerosis were at low or moderate risk. In Table 5, we presented the correlation between the cardiovascular risk level with the presence or not of carotid atherosclerosis.

\section{Discussion}

It is currently well documented that cardiovascular risk is increased in autoim- 
mune diseases. Ischemic complications are thought to be the first cause of mortality after 5 years of SLE progression [1]. But evidence from the literature suggests Table 5. Prediction of cardiovascular risk by the classical equations and their modified forms compared to the vascular ultrasound.

\begin{tabular}{lccc}
\hline \multirow{2}{*}{ Cardiovascular risk level } & \multicolumn{3}{c}{ Carotid ultrasound } \\
\cline { 2 - 4 } Framingham (\%) & Without ATH * $\mathbf{n}(\%)$ & With $\mathrm{ATH}^{*} \mathrm{n}(\%)$ & Total (\%) \\
Moderate/low & $40(97.4)$ & $6(75)$ & $46(93.6)$ \\
Very high/high & $1(2.6)$ & $2(25)$ & $3(6.4)$ \\
Framingham/m (\%) & & & \\
Moderate/low & $37(90)$ & $4(50)$ & $41(83.3)$ \\
Very high/high & $4(10)$ & $4(50)$ & $8(16.7)$ \\
SCORE (\%) & & & \\
Moderate/low & $41(100)$ & $8(100)$ & $49(100)$ \\
Very high/high & $0(0)$ & $0(0)$ & $0(0)$ \\
SCORE/m (\%) & & $7(87.5)$ & $48(97.9)$ \\
Moderate/low & $41(100)$ & $1(12.5)$ & $1(2.1)$ \\
Very high/high & $0(0)$ & $8(100)$ & $49(100)$ \\
Total (\%) & $41(100)$ & &
\end{tabular}

ATH $=$ atherosclerosis

$\begin{array}{llll}\text { Framingham } & \text { Framingham } / \mathrm{m} & \text { SCORE } & \text { SCORE } / \mathrm{m} \\ \text { Sensitivity }=25 \% & \text { Sensitivity }=50 \% & \text { Sensitivity }=0 \% & \text { Sensitivity }=12.5 \% \\ \text { Specificity }=97 \% & \text { Specificity }=90 \% & \text { Specificity }=100 \% & \text { Specificity }=100 \%\end{array}$

that in SLE, the equations classically use for predicting cardiovascular risk are not suitable because they underestimate this risk [2]. In the general population, Framingham and SCORE equations have been validated at the individual level for predicting cardiovascular risk [4] [5]. Cardiovascular risk may be more widespread in Africa where access to care is more limited and screening for atherosclerosis is less easy and less systematic [12]. However, it remains little studied in sub-Saharan Africa, some publications concern rheumatoid arthritis [13] [14] [15]. It is in this context that we conducted a cross-sectional study to assess the performance of equations in predicting this cardiovascular risk during SLE compared to carotid ultrasound in defining high risk. Carotid ultrasound was used for its greater accessibility and safety. Also, this methodology has been used in several previous studies [16] [17].

The increased cardiovascular risk in SLE is partly due to the higher frequency of traditional factors in this population [18]. In our study, dyslipidemia was present in more than half of the cases. It concerned a low-HDL-cholesterolemia and a high triglyceridemia. Similarly, almost half of the patients had hyperuricemia. Similar results have been reported in the literature [17] [19]. 
Carotid atherosclerosis was noted in $16 \%$ of patients. In the meta-analysis of SLE and atherosclerosis, 44 out of 71 studies found a higher prevalence of plaques in SLE cases than in controls (23.6\% versus 13\%) [17].

Cardiovascular complications are an important mortality factor in SLE. So, their early detection is a necessary element in the management of this disease. Similarly, the assessment of cardiovascular risk appears to be important in the prevention of these complications.

In SLE, earlier atherosclerosis is due to high prevalence of traditional factors but also disease related factors. That is why conventional equations underestimate the cardiovascular risk in SLE because they don't consider these specifics factors [2] [20] [21]. The Framingham score is heavily weighted for age and male sex, so the risk of coronary heart disease in young women with SLE is grossly underestimated. The inability to accurately assess the true risk of cardiovascular disease for each individual patient has resulted in an inability to modify mortality associated with cardiovascular events and morbidity in LSE, in contrast to the trend observed for other causes of mortality, such as lupus nephritis [22]. In our study, the cardiovascular risk is underestimated by the conventional equations (Framingham and SCORE) compared to the carotid ultrasound. The Hopkins Lupus Cohort Study compared the prediction of major cardiovascular events between the Framingham equation, the American College of Cardiology (AHA/ACC) equation and a formula that considered SLE factors and traditional risk factors. This study concluded that the Hopkins lupus cohort formula was superior to the Framingham and AHA/ACC equations [23]. In the retrospective series by Esdaile et al., the observed coronary event rate was 7.5 times (95\% CI: 5.1 - 10.4) higher than expected based on the Framingham score, while the ischemic stroke rate was 7 times (95\% CI: 4.0 - 13.6) higher, with an absolute risk of coronary events of $12.9 \%$ ( $1.5 \%$ per year) and ischemic strokes of $6.1 \%$ ( $0.70 \%$ per year), after a mean follow-up of 8.6 years [20]. Kawai et al. found no significant difference in the level of cardiovascular assessed by the Framingham score between SLE cases and controls. Furthermore, in this study, the Framingham cardiovascular level of lupus patients did not predict the existence of subclinical atherosclerosis detected by coronary calcifications [24]. Urowitz proposed an adjustment of the Framingham score by a factor of 1.5, 2 and 3. Thus, he found that a factor of 2 improved the sensitivity with good specificity of the Framingham score [25].

After adjustment (multiplying by a factor of 1.5), we find that the modified Framingham and SCORE equation still have a low sensitivity, respectively 50\% and $12.5 \%$, compared to carotid vascular ultrasound.

A recent study assessed the performance of eight clinical risk prediction scores (including Framingham, SCORE and their modified forms) to identify individuals with SLE at high cardiovascular disease risk, as defined by the presence of atherosclerotic plaques. In this study, most of the five generic and three "SLE-adapted" clinical risk scores underestimated high cardiovascular risk defined by atheroscle- 
rotic plaque presence in patients with SLE [26].

Our work is limited by the small sample size and cross-sectional nature of the study. A prospective study to determine the occurrence of cardiovascular events would better assess the performance of these equations.

\section{Conclusion}

Most of our study population was classified in low cardiovascular according to the Framingham, SCORE and their modified forms of risk equations. These equations had a poor predictive value of high cardiovascular compared to the carotid ultrasound (sensitivity $\leq 50 \%$ ). The modified Framingham offered the best sensitivity of $50 \%$ for LS.

\section{Conflicts of Interest}

The authors declare no conflicts of interest regarding the publication of this paper.

\section{References}

[1] Borchers, A.T., Naguwa, S.M. and Shoenfeld, Y. (2010) The Geoepidemiology of Systemic Lupus Erythematosus. Autoimmunity Reviews, 9, A277-A287. https://doi.org/10.1016/j.autrev.2009.12.008

[2] Arnaud, L., Mathian, A. and Bruckert, E. (2014) Le risque cardiovasculaire au cours du lupus systémique. La revue de médecine interne, 35, 723-729. https://doi.org/10.1016/j.revmed.2014.07.005

[3] Serraj, K., Mecili, M. and Zahi, I. (2011) Connectivites et risque cardiovasculaire: Etat des lieux. Revue médecine thérapeutique, 17, 11-22.

[4] Conroy, R.M., Pyrölä, K., Fitzgerald, A.P., et al. (2003) Estimation of 10-Year Risk of Fatal Cardiovascular Disease in Europe: The SCORE Project. European Heart Journal, 24, 987-1003. https://doi.org/10.1016/S0195-668X(03)00114-3

[5] Wilson, P.W., D’Agostino, R.B., Levy, D., et al. (1998) Prediction of Coronary Heart Disease Using Risk Factor Categories. Circulation, 97, 1837-1834. https://doi.org/10.1161/01.CIR.97.18.1837

[6] Roman, M.J., Naqvi, T.Z., Gardin, J.M., et al. (2006) Clinical Application of Noninvasive Vascular Ultrasound in Cardiovascular Risk Stratification: A Report from the American Society of Echocardiography and the Society of Vascular Medicine and Biology. Journal of the American Society of Echocardiography, 19, 943-954. https://doi.org/10.1016/j.echo.2006.04.020

[7] Zeller, C.B. and Appenzeller, S. (2008) Cardiovascular Disease in Systemic Lupus Erythematosus: The Role of Traditional and Lupus Related Risk Factors. Current Cardiology Reviews, 4, 116-122. https://doi.org/10.2174/157340308784245775

[8] Alenghata, F.J. (2016) The Prevalence of Atherosclerosis in Those with Inflammatory Connective Tissue Disease by Race, Age, and Traditional Risk Factors. Scientific Reports, 6, Article No. 20303. https://doi.org/10.1038/srep20303

[9] Hochberg, M.C. (1997) Updating the American College of Rheumatology Revised Criteria for the Classification of Systemic Lupus Erythematosus. Arthritis \& Rheumatology, 40, 17-25. https://doi.org/10.1002/art.1780400928 
[10] Parodis, I., Emamikia, S., Gomez, A., et al. (2018) Clinical SLEDAI-2K Zero May Be a Pragmatic Outcome Measure in SLE Studies. Expert Opinion on Biological Therapy, 19, 157-168. https://doi.org/10.1080/14712598.2019.1561856

[11] Corrales, A., González-Juanatey, C., Peiró, M.E., et al. (2014) Carotid Ultrasound Is Useful for the Cardiovascular Risk Stratification of Patients with Rheumatoid Arthritis: Results of a Population-Based Study. Annals of the Rheumatic Diseases, 73, 722-727. https://doi.org/10.1136/annrheumdis-2012-203101

[12] Meyer, O. (2002) Lupus systémique chez les non-Caucasiens. Revue du Rhumatisme, 69, 801-808. https://doi.org/10.1016/S1169-8330(02)00385-X

[13] Dessein, P.H., Semb, A.G., González-Gay, M.A., et al. (2015) Enhanced Cardiovascular Risk in Rheumatoid Arthritis: Elucidation, Assessment, and Management. BioMed Research International, 2015, Article ID: 850671. https://doi.org/10.1155/2015/850671

[14] Mulumba, C., Lebughe, P., Mbuyi-Muamba, J.M., et al. (2019) Prevalence and Associated Factors of Subclinical Atherosclerosis in Rheumatoid Arthritis at the University Hospital of Kinshasa. BMC Rheumatology, 3, 37-59.

https://doi.org/10.1186/s41927-019-0085-4

[15] Solomon, A., Stanwix, A.E., Castañeda, S., et al. (2020) Points to Consider in Cardiovascular Disease Risk Management among Patients with Rheumatoid Arthritis Living in South Africa, an Unequal Middle Income Country. BMC Rheumatology, 4, 42-88. https://doi.org/10.1186/s41927-020-00139-2

[16] Dessein, P.H., Corrales, A., Lopez-Mejias, R., et al. (2016) The Framingham Score and the Systematic Coronary Risk Evaluation at Low Cutoff Values Are Useful Surrogate Markers of High-Risk Subclinical Atherosclerosis in Patients with Rheumatoid Arthritis. The Journal of Rheumatology, 43, 486-494.

https://doi.org/10.3899/jrheum.150510

[17] Wu, G.C., Liu, H.R., Leng, R.X., et al. (2015) Subclinical Atherosclerosis in Patients with Systemic Lupus Erythematosus: A Systemic Review and Meta-Analysis. $\mathrm{Au}$ toimmunity Reviews, 15, 22-37. https://doi.org/10.1016/j.autrev.2015.10.002

[18] Liu, Y. and Kaplan, M.J. (2018) Cardiovascular Disease in Systemic Lupus Erythematosus: An Update. Current Opinion in Rheumatology, 30, 441-448. https://doi.org/10.1097/BOR.0000000000000528

[19] Park, J.K., Kim, J.Y., Moon, J.Y., et al. (2016) Altered Lipoproteins in Patients with Systemic Lupus Erythematosus Are Associated with Augmented Oxidative Stress: A Potential Role in Atherosclerosis. Arthritis Research \& Therapy, 18, 306-330. https://doi.org/10.1186/s13075-016-1204-x

[20] Esdaile, J.M., Abrahamowicz, M., Grodzicky, T., et al. (2001) Traditional Framingham Risk Factors Fail to Fully Account for Accelerated Atherosclerosis in Systemic Lupus Erythematosus. Arthritis \& Rheumatology, 44, 2331-2337. https://doi.org/10.1002/1529-0131(200110)44:10<2331::AID-ART395>3.0.CO;2-I

[21] Urowitz, M.B., Ibanez, D., Su, J., et al. (2016) Modified Framingham Scores for Systemic Lupus Erythematosus. The Journal of Rheumatology, 43, 875-879. https://doi.org/10.3899/jrheum.150983

[22] Croca, S. and Rahman, A. (2017) Atherosclerosis in Systemic Lupus Erythematosus. Best Practice \& Research: Clinical Rheumatology, 31, 364-372.

https://doi.org/10.1016/j.berh.2017.09.012

[23] Petri, M.A., Barr, E. and Magder, L.S. (2019) Development of a Systemic Lupus Erythematosus Cardiovascular Risk Equation. Lupus Science \& Medicine, 6, e000346. https://doi.org/10.1136/lupus-2019-000346 
[24] Kawai, V.K., Solus, J.F., Oeser, A., et al. (2011) Novel Cardiovascular Risk Prediction Models in Patients with Systemic Lupus Erythematosus. Lupus, 20, 1526-1534. https://doi.org/10.1177/0961203311420317

[25] Urowitz, M.B., Ibanez, D. and Gladman, D.D. (2012) Systemic Lupus International Collaborating Clinics (SLICC). Adjusted Framingham Risk Factor Scoring for Systemic Lupus Erythematosus: Results from an Inception Cohort Followed for Eight Years. Arthritis \& Rheumatology, 64, 647.

[26] Drosos, G.C., Konstantonis, G., Sfikakis, P.P. and Tektonidou, M.G. (2020) Underperformance of Clinical Risk Scores in Identifying Vascular Ultrasound-Based High Cardiovascular Risk in Systemic Lupus Erythematosus. European Journal of Preventive Cardiology, 28, 346-352. https://doi.org/10.1177/2047487320906650 